\title{
Implementation of a Unimodularity Test
}

\author{
Matthias Walter ${ }^{1}$ and Klaus Truemper ${ }^{2}$ \\ ${ }^{1}$ Institute of Mathematical Optimization, \\ University of Magdeburg "Otto von Guericke", \\ 39106 Magdeburg, Germany \\ ${ }^{2}$ Department of Computer Science, \\ University of Texas at Dallas, \\ Richardson, TX 75083, U.S.A.
}

\begin{abstract}
This paper describes implementation and computational results of a polynomial test of total unimodularity. The test is a simplified version of a prior method. The program also decides two related unimodularity properties. The software is available free of charge in source code form under the Boost Software License.
\end{abstract}

Keywords: unimodularity, total unimodularity, polynomial test

\section{Introduction}

This paper describes the implementation of a simplified version of the polynomial test 17] for total unimodularity. The program also decides absence/presence of two related types of unimodularity. The computer program is available free of charge in source code from two sites [14] under the Boost Software License [3]. Computational effectiveness is demonstrated for nontrivial test instances.

We begin with a well-known definition. An integer matrix $A$ is totally unimodular (t.u.) if every square submatrix $D$ of $A$ has $\operatorname{det} D=0$ or \pm 1 . This property was introduced by [10] with slightly different terminology. The reference establishes a key result for t.u. matrices: The inequality $A x \leq b$ has all basic solutions integer for all integer vectors $b$ if and only if $A$ is t.u.

Several other concepts are closely related to total unimodularity; see, for example, 11 15 20|16. Here, we cover the following two properties. An integer matrix $A$ is unimodular if for every column basis $C$ of $A$, the maximal square submatrices $C^{i}$ of $C$ satisfy $\operatorname{gcd}_{i} \operatorname{det} C^{i}=1$. In the special case of unimodularity where each $C^{i}$ has $\operatorname{det} C^{i}=0$ or \pm 1 , the matrix $A$ is strongly unimodular. Analogously to the key result for t.u. matrices, the equation $A x=b$ has all basic solutions integer for all integer vectors $b$ if and only if $A$ is unimodular. The next theorem is taken from [15. 
Theorem 1. Let $A$ be an integer matrix.

1. A is unimodular if and only if, for an arbitrarily selected column basis matrix $C$ of $A, \operatorname{gcd}_{i} \operatorname{det} C^{i}=1$ and the solution $X$ of $C X=A$ is t.u.

2. $A$ is strongly unimodular if and only if both $A$ and its transpose $A^{t}$ are unimodular.

The question whether a given column basis $C$ of an integer matrix $A$ satisfies $\operatorname{gcd}_{i} \operatorname{det} C^{i}=1$ can be efficiently answered via the Smith Normal Form of [13. Thus, testing for any of the above properties is readily reduced to testing for total unimodularity. The software carries out the same reductions. We skip implementation details and focus on the test of total unimodularity.

Let $A$ be a given integer matrix and $B$ be the binary matrix derived from $A$ by converting each -1 to 1 . Define $I$ to be the identity of appropriate order. It is well known that the following four steps decide absence/presence of total unimodularity.

In the first step, a trivial check verifies that all nonzeros of $A$ are 1 or -1 . Clearly, $A$ is not t.u. if the matrix fails this test. In the second step, it is checked whether the binary matroid $M(B)$ represented by the matrix $[I \mid B]$ has the property of regularity defined by [21]. For our purposes, it is convenient to declare $M(B)$ to be regular if $B$ can be signed to become t.u. Testing for regularity of $M(B)$ is the most difficult step. If the answer is negative, then $A$ cannot be t.u. So assume that $M(B)$ is regular. In the third step, suitable signing of the $1 \mathrm{~s}$ of $B$ converts that matrix to a t.u. matrix $A^{\prime}$. This step is based on the uniqueness of such signing, up to scaling, proved by [4]. The process is quite straightforward. The uniqueness result of [4] is once more employed in the fourth step, where it is checked whether $A^{\prime}$ can by column and row scaling be converted to $A$. This test is very easy. The matrix $A$ is t.u. if and only if such scaling is possible.

As far as we know, every prior polynomial algorithm for testing matroid regularity uses the regular matroid decomposition of [12]. Indeed, implicit in the cited reference is already one such scheme, provided the proofs are implemented in suitable algorithmic steps. Of the prior methods [215]17, the scheme of [17] has lowest order, which for a binary $m \times n$ matrix is $O\left((m+n)^{3}\right)$. We use the latter method as basis for the implementation. We say "as basis" since, for a first attempt, full implementation of all features of [17] was rather daunting. Hence, we opted for a simplified version that avoids complex operations but still is in the spirit of the method. The next section summarizes that implementation while pointing out differences to [17]. Section 3 introduces two naïve enumerative tests for comparison purposes. Section 4 describes computational results for nontrivial test instances. Section 5 contains technical details of the implementation.

\section{Summary of Implementation}

This section describes the implementation and compares it with the method of [17]. For an abbreviated terminology, implemented method refers to the method implemented to-date, while original method is the scheme of the cited reference. 
We strive for an intuitive discussion so that salient ideas emerge and a clutter of technical details is avoided. The reader not familiar with matroid theory may want to rely on the introductory discussion of binary, graphic, and regular matroids of [19] before proceeding.

We first review key concepts for testing matroid regularity. For details, see 12]19.

\subsection{Key Concepts}

We begin with some definitions regarding matrix notation. Let $A$ be a matrix whose rows (resp. columns) are indexed by a set $Z$ (resp. $Y$ ). For any subsets $Z^{\prime} \subseteq Z$ and $Y^{\prime} \subseteq Y$, the submatrix of $A$ indexed by $Z^{\prime}$ and $Y^{\prime}$ is denoted by $A_{Z^{\prime}, Y^{\prime}}$. If $Z^{\prime}$ is a singleton set, say consisting of an element $z$, then we use just $z$ instead of $\{z\}$ in the above notation. A singleton set containing an element $y$ of $Y$ is handled analogously. In particular, $A_{z, y}$ is the entry of $A$ indexed by $z$ and $y$. The abbreviated notation is not ambiguous since we always employ upper-case letters for sets and lower-case letters for elements of sets.

For a binary matrix $[I \mid B]$, let $X$ and $Y$ index the columns of the submatrices $I$ and $B$, respectively. The matroid $M(B)$ has $X \cup Y$ as groundset. For $X^{\prime} \subseteq X$ and $Y^{\prime} \subseteq Y$, the subset $X^{\prime} \cup Y^{\prime}$ of $X \cup Y$ is independent in the matroid if the column submatrix of $[I \mid B]$ indexed by $X^{\prime} \cup Y^{\prime}$ has linearly independent columns. We avoid explicit display of the matrix $I$ by indexing the rows of $B$ by $X$, in addition to the column index set $Y$. Then $X^{\prime} \cup Y^{\prime}$ is independent in $M(B)$ if and only if the submatrix $B^{\prime}=B_{X \backslash X^{\prime}, Y^{\prime}}$ has independent columns.

We consider two matrices equal if they become numerically the same under suitable row and column permutations. The indices of rows and columns are ignored in the comparison. It is convenient that we apply matroid terminology for $M(B)$ to $B$ as well. Thus, $B$ is regular if $M(B)$ has that property, that is, if $B$ can be signed to become a t.u. matrix.

For $x \in X$ and $y \in Y$, a pivot on a nonzero entry $B_{x, y}$ of the matrix $[I \mid B]$ is the customary set of elementary row operations. In the reduced notation where $I$ is not explicitly listed, the pivot converts $B$ to a matrix $B^{\prime}$ that agrees numerically with $B$ except for the entries $B_{i, j}^{\prime}$ where $i \neq x, j \neq y$, and $B_{x, j}=B_{i, y}=1$. The matrix $B^{\prime}$ has the same index sets as $B$ except that the indices $x$ and $y$ have traded places. The matrix $B$ is regular if and only if this holds for $B^{\prime}$.

$M(B)$ and $B$ are graphic if there exists an undirected graph $G$ with edges indexed by the elements of $X \cup Y$ such that the edge sets of subgraphs of $G$ without any cycle are precisely the independent sets of $M(B)$. Note that each zero column of $B$ corresponds to a loop of $G . M(B)$ and $B$ are cographic if the transpose of $B$, denoted by $B^{t}$, is graphic. $M(B)$ and $B$ are planar if $B$ is graphic and cographic.

A graphic $B$ is regular. Since $B$ can be signed to become t.u. if and only if this is so for $B^{t}$, a cographic $B$ is regular as well. There exist very fast algorithms for deciding whether $B$ is graphic [17].

Define $B G(B)$ to be the bipartite graph with node set $X \cup Y$ where an undirected edge joins nodes $x \in X$ and $y \in Y$ if $B_{x, y}=1$. Declare the matrix 
$B$ to be connected if the graph $B G(B)$ is connected. Define the length of $B$, denoted by $s(B)$, to be the number of rows plus the number of columns of $B$. We allow matrices to have no rows or columns. The rank of any such matrix is 0 .

If $B$ has zero or unit vector rows or columns, or has duplicate rows or columns, then recursive deletion of zero/unit vectors and of duplicates except for representatives, reduces $B$ to a simple matrix $B^{\prime}$. The matrix $B$ is regular if and only if this is so for $B^{\prime}$. If $B^{\prime}$ has no rows or columns, then $B$ is regular. For the definitions to follow, we assume that $B$ is simple.

If matrix $B$ has block structure, say with blocks $B^{i}, i=1,2, \ldots$, then $B$ is a 1-sum of the blocks $B^{i}$, and $B$ is regular if and only if each of the blocks $B^{i}$ has that property. The implemented method detects blocks via $B G(B)$. For the remaining discussion of this section, we assume that the simple $B$ has no such block structure, which is equivalent to assuming that $B$ is connected.

Suppose $B$ has the form

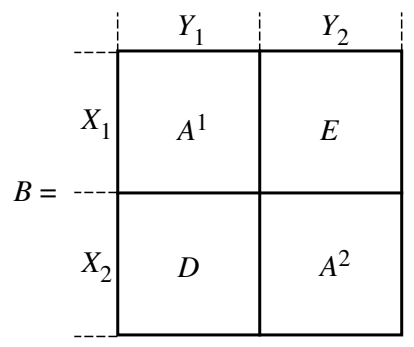

Fig. 1. Separation of $B$

Define $k=\operatorname{rank}(D)+\operatorname{rank}(E)+1$. If the lengths of $A^{1}$ and $A^{2}$ satisfy $s\left(A^{1}\right) \geq k$ and $s\left(A^{2}\right) \geq k$, then $B$ has a $k$-separation. If either $s\left(A^{1}\right)=k-1$ and $s\left(A^{2}\right) \geq k$, or $s\left(A^{1}\right) \geq k$ and $s\left(A^{2}\right)=k-1$, then $B$ has a deficient $k$-separation. If, for some $l \geq k, s\left(A^{1}\right) \geq l$ and $s\left(A^{2}\right) \geq l$, then $B$ has a $(k \mid l)$-separation.

If the submatrix $E$ of $B$ is nonzero, then by pivots in $E$ we can always obtain a matrix $B^{\prime}$ with the same type of separation where $E^{\prime}=0$ and $\operatorname{rank}\left(D^{\prime}\right)=k-1$. In the discussion below, we assume that $B$ itself is of that form.

In the case of a 2-separation, the submatrix $D$ of Fig. 1 has rank equal to 1 , and $B$ has the following form. 


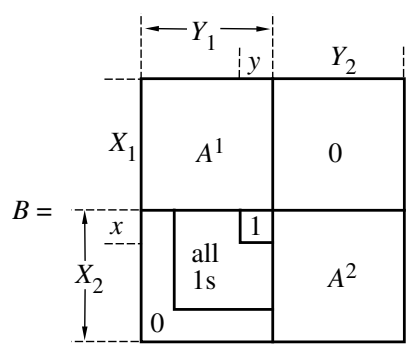

Fig. 2. 2-Sum Case

If in addition both $A^{1}$ and $A^{2}$ have at least one entry, then $B$ is a 2 -sum with the following component matrices $B^{1}$ and $B^{2}$.
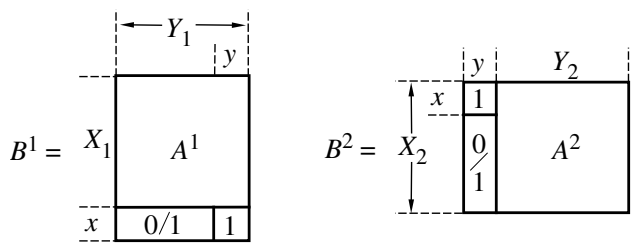

Fig. 3. 2-sum Components

Let $\bar{B}$ be the submatrix $B_{x, y}$ of $B$ in Fig. 2 The same submatrix occurs in $B^{1}$ and $B^{2}$ of Fig. 3. That submatrix has rank equal to 1 . It is called the connecting submatrix of the 2 -sum decomposition. When $B^{1}$ and $B^{2}$ are overlaid such that the two connecting submatrices are identified, then straightforward computations produce the matrix $B$ of Fig. 22, see Chapter 8 of [19]. The matrix $B$ of Fig. 2 is regular if and only if this is so for $B^{1}$ and $B^{2}$.

Continuing the discussion of matrix features, suppose that $B$ is connected, simple, and has no 2-sum decomposition. Such a matrix is called 3-connected. Assume that a 3 -connected $B$ has a $(3 \mid l)$-separation where $l \geq 4$. It is not difficult to prove that pivots can produce in $B$ the following structure for the submatrices $A^{1}, A^{2}$, and $D$, where $\bar{D}$ has the same rank as $D$, that is, 2 . 


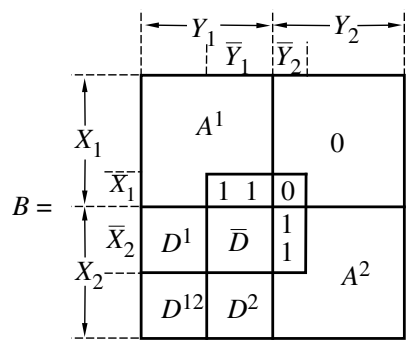

Fig. 4. 3-sum Case

Then $B$ is a 3 -sum with the following component matrices $B^{1}$ and $B^{2}$.
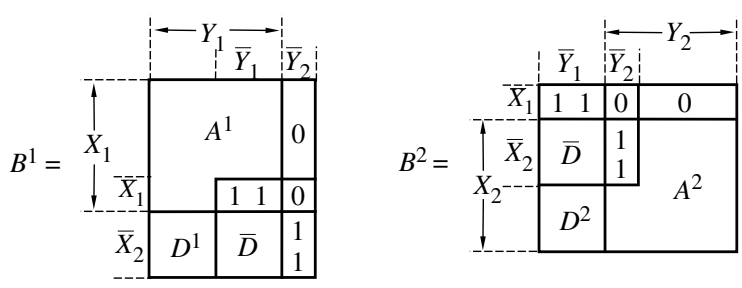

Fig. 5. 3-sum Components

For $B$ of Fig. 4 define $\bar{B}$ to be the submatrix $B_{\bar{X}_{1} \cup \bar{X}_{2}, \bar{Y}_{1} \cup \bar{Y}_{2}}$. The same submatrix occurs in $B^{1}$ and $B^{2}$ of Fig. 5 . Evidently, $\bar{B}$ is a $3 \times 3$ matrix, so $\bar{D}$ is a $2 \times 2$ matrix. Since $\bar{D}$ has rank equal to 2 , it must be an identity matrix or have exactly three $1 \mathrm{~s}$. The matrix $\bar{B}$ is the connecting submatrix of the 3 -sum decomposition. It is easy to check that $\bar{B}$ is graphic. The corresponding graph is the wheel $W_{3}$ with three spokes. When $B^{1}$ and $B^{2}$ are overlaid such that the two connecting submatrices are identified, then, analogously to the 2-sum case, straightforward computations produce the matrix $B$ of Fig. 4

The matrix $B$ of Fig. 4 is regular if and only if this is so for $B^{1}$ and $B^{2}$ of Fig. 5 The above 2 -sums and 3 -sums decompositions can be found by the matroid intersection algorithm of [6] plus some pivots.

Finally, there is a regular matroid on 10 elements called $R_{10}$. There are only two matrices that represent $R_{10}$. They are $B^{10.1}$ and $B^{10.2}$ below. 

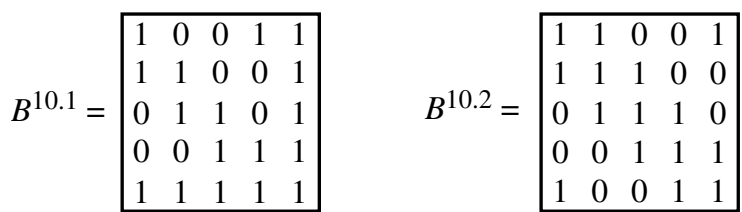

Fig. 6. Matrices $B^{10.1}$ and $B^{10.2}$ for $R_{10}$

$R_{10}$ is the smallest regular matroid that is not graphic and not cographic. It is simple, connected, and does not have a 2- or 3-sum decomposition.

In slightly different form and distributed among a number of references, the concepts and ideas stated above were known prior to 1978. But that knowledge was not sufficient to establish a polynomial testing algorithm for regularity. That situation changed in 1978 when Seymour constructed the decomposition theorem for the regular matroids [12, which supports efficient testing of regularity. Indeed, the cited reference implicitly already contains such a scheme, provided certain nonconstructive proofs are replaced by constructive ones involving polynomial subroutines. A simplified version of the theorem that suffices for present purposes is stated next.

Theorem 2. For any regular matrix, at least one of the statements (i)-(vi) applies.

(i) $B$ is graphic or cographic.

(ii) B has a zero or unit vector row or column, or has duplicate rows or columns.

(iii) $B$ is simple and a 1-sum.

(iv) $B$ is simple, connected, and a 2-sum.

(v) $B$ is 3-connected and, after suitable pivots, has the form of a 3-sum that corresponds to a (3|6)-separation. Let $\bar{B}$ with row index set $\bar{X}$ and column index set $\bar{Y}$ be any 3 -connected nongraphic and noncographic submatrix of $B$. Then there is a (3|6)-separation of $B$, say defined by index sets $X_{1}, X_{2}, Y_{1}$, and $Y_{2}$, such that $X_{1} \cap \bar{X}, X_{2} \cap \bar{X}, Y_{1} \cap \bar{Y}$, and $Y_{2} \cap \bar{Y}$ define a (3|6)-separation of $\bar{B}$.

(vi) $B$ is equal to $B^{10.1}$ or $B^{10.2}$ of Fig. 6 .

Theorem 2 supports the following polynomial algorithm for testing regularity. Given a matrix $B$, check with one of the methods of [1] whether $B$ or $B^{t}$ is graphic. If this is so, $B$ has been proved to be regular. Otherwise, reduce $B$ to a simple matrix and check if it has a 1-, 2-, or 3-sum decomposition, in that order, using the graph $B G(B)$ for the 1-sum case and the matroid intersection algorithm of [6] for the 2- and 3- sum cases. If a decomposition is detected, carry out the decomposition and apply the algorithm recursively to the components. Otherwise, check if $B$ is equal to one of the matrices $B^{10.1}$ and $B^{10.1}$ of Fig. 6. If this is the case, $B$ is regular. Otherwise, declare $B$ to be nonregular.

References [25] describe sophisticated versions of the above approach, with bound $\left.O\left((m+n)^{4.5}\right)(\log (m+n))^{0.5}\right)$ for [2] and $O\left((m+n)^{5}\right)$ for [5]. A lower 
complexity can be achieved when the tests of graphicness and the search for decompositions are intertwined, and when the latter search is carried out by a certain induced decomposition scheme instead of the matroid intersection algorithm [6]. This is done in [17], producing a test with bound $O\left((m+n)^{3}\right)$. The next section gives insight into that algorithm, which according to the convention introduced earlier is called the original method. In the description below, the emphasis is on providing intuitive insight into the method instead of a mathematically precise specification, which is included in [17.

\subsection{Original Method}

The method initializes a set $\mathcal{B}$ with a matrix $B_{0}$ that is to be tested for regularity. The method removes (resp. adds) matrices from (resp. to) $\mathcal{B}$ until $\mathcal{B}$ becomes empty or nonregularity of some matrix in $\mathcal{B}$ has been proved. In the former (resp. latter) case, $B$ has been proved to be regular (resp. not regular). For some matrices of $\mathcal{B}$, additional information is recorded. Details are covered in the description of the method.

0 . If $\mathcal{B}$ is empty, declare the initial matrix $B_{0}$ to be regular, and stop. Otherwise remove an arbitrary matrix $B$ from $\mathcal{B}$. If $B$ is known to be 3-connected, go to Step 3.

1. If $B$ is not simple, remove zero and unit vectors and reduce duplicate vectors to representatives. If $B$ is not connected, carry out a 1-sum decomposition via the graph $B G(B)$, place the components into $\mathcal{B}$, and go to Step 0 .

2. Determine a sequence of nested 3-connected submatrices $N^{1}, \ldots, N^{k}$ for some $k \geq 1$, where (1) $N^{1}$ is graphic and the corresponding graph $G$ is the wheel $W_{3},(2)$ for each $i>1, N^{i}$ contains $N^{i-1}$ as proper submatrix and the lengths of $N^{i}$ and $N^{i-1}$ satisfy $s\left(N^{i}\right) \leq s\left(N^{i-1}\right)+3$. We skip detailed discussion of the steps finding such a sequence. They are described in procedure FIND-B and EXTEND-B of the original method, except that FIND-B or EXTEND-B assume 3-connectedness of $B$ and can be trivially modified to detect 2-sum decompositions. Suffice it to say here that the steps make repeated use of breadth-first-search (BFS) in the graph $B G(B)$ or a closely related graph, and of certain path shortening pivots. If the modified FIND-B or EXTEND$\mathrm{B}$ detects a 2 -sum decomposition, place the components of the 2-sum into $\mathcal{B}$ and return to Step 0. Otherwise, the last matrix $N^{k}$ of the sequence of nested 3 -connected submatrices is equal to $B$. Place $B$ into $\mathcal{B}$, suitably record with it the 3-connected extension sequence, and go to Step 0.

3. If $B$ is not supplied with a sequence of nested 3-connected submatrices, construct such a sequence as described in Step 2, except that the 2-sum decomposition case cannot occur. Regardless of the case, each 3-connected submatrix $N^{i}$ triggers additional testing as described next. Recall that $N^{1}$ is graphic, indeed represents the wheel $W_{3}$. It is assumed inductively that $N^{i-1}$ is graphic and possibly planar.

4. If $N^{i-1}$ is planar, check whether $N^{i}$ is planar. If the answer is negative, check if $N^{i}$ is graphic or cographic; if $B$ turns out to be cographic, apply the 
transpose operator to $B$ and its submatrices. Thus, there are three possible outcomes: $N^{i}$ is planar, or graphic but not cographic, or not graphic and not cographic. The test is carried out by TEST-C of the original method. It is very efficient due a key result of 22] according to which a 3-connected graphic matrix has exactly one corresponding graph. If $N^{i}=B$ and $N^{i}$ is planar or graphic, then $B$ is regular; go to Step 0 .

5. Determine whether any one of certain $(3 \mid l)$-separations, $l \geq 3$, of $N^{i}$ can be extended to a $\left(3 \mid l^{\prime}\right)$-separation of $B$ for $l^{\prime} \geq 4$. If that is so, the latter separation is induced by the former one. The test uses the straightforward subroutine PARTITION of the original method, but nevertheless is rather complicated since it exploits specific structural information concerning $N^{i-1}$ and $N^{i}$. If an induced $\left(3 \mid l^{\prime}\right)$-separation is found, determine the corresponding 3 -sum decomposition, place the components into $\mathcal{B}$, and go to Step 0 . In a rather complex process, retain additional information that concerns the current sequence of nested 3-connected submatrices and, for special cases, facts about induced decompositions. The retained information is used later when the component is removed from $\mathcal{B}$ and processed. It is precisely this carryover of information that makes the relatively low complexity of the original method possible. If no induced $\left(3 \mid l^{\prime}\right)$-separation is found, proceed as follows depending on the classification of $N^{i}$ of Step 4: (1) If $N^{i}$ is graphic, return to Step 2 to extend the current sequence of nested 3-connected submatrices.

(2) If $N^{i}$ is not graphic, equal to $B$, and equal to one of $B^{10.1}$ or $B^{10.2}$, then go to Step 0. (3) Otherwise, $N^{i}$ is not regular; declare that $B$ of the original $\mathcal{B}$ is not regular, and stop.

\subsection{Implemented Method}

The main difference between the original and the implemented method is replacement of the complicated Step 5 by a simpler, enumerating search that looks for induced (3|4)-separations. The search is started when (1) a 3-connected matrix $B$ not equal to $B^{10.1}$ or $B^{10.2}$ is at hand; (2) a sequence of 3 -connected nested $N^{1}, \ldots, N^{k}=B$ has been found where the length of $N^{1}$ satisfies $8 \leq s\left(N^{1}\right) \leq 10$, and where for $2 \leq i \leq k$, the lengths of $N^{i}$ and $N^{i-1}$ satisfy $s\left(N^{i}\right) \leq s\left(N^{i-1}\right)+3$; and (3) for some $1 \leq j<k$ and all $1 \leq i \leq j$, the matrices $N^{i}$ are graphic or cographic, while $N^{j+1}$ is not graphic and not cographic. Let $E^{i}$ be the union of the row and column index sets of the matrices $N^{i}$ of the sequence.

Since $N^{j+1}$ is not graphic and not cographic, $B$ is not graphic and not cographic. If $B$ is regular, then by Theorem 2(v) there is a (3|6)-separation of $B$ that can be reduced to a $(3 \mid 6)$-separation of $N^{j+1}$ by suitable reduction of the index sets of the separation. Since $s\left(N^{i}\right) \leq s\left(N^{i-1}\right)+3$, the matrix $N^{j}$ has a 3 -separation that induces the (3|6)-separation of $B$. In the search described next, we look instead for a less demanding (3|4)-separation of $B$ induced by a possibly deficient 3-separation of one of the matrices $N^{1}, \ldots, N^{j}$.

We first generate all pairs $\left(T, E^{1} \backslash T\right)$ where (1) $T$ satisfies $|T| \leq\left|E^{1} \backslash T\right|$, and (2) $X_{1}=X \cap T, X_{2}=X \backslash X_{1}, Y_{1}=Y \cap T$, and $Y_{2}=Y \backslash Y_{1}$ define a possibly deficient 3-separation of $N^{1}$. The number of such pairs is bounded by a constant 
since $\left|E^{1}\right| \leq 10$. For $i=2, \ldots, j$, we then generate all pairs $\left(T, T \backslash E^{i}\right)$ where (1) $T$ contains at least one element of $E^{i} \backslash E^{i-1}$ and at most one element of $E^{i-1}$, and (2) the pair defines a possibly deficient 3-separation of $N^{i}$ analogously to the $N^{1}$ case. Thus, the number of pairs for case $i$ is linear in $\left|E^{i}\right|$, and overall a total of $O\left((m+n)^{2}\right)$ pairs are produced.

The construction rules of the pairs obtained from the sequence $N^{1}, \ldots, N^{j}$ assure validity of the following claim. If $B$ is regular, then, for some $i \leq j$, the possibly deficient 3 -separation of $N^{i}$ corresponding to one of the derived pairs induces a (3|4)-separation of $B$.

We use PARTITION of the original method to derive such an induced (3|4)separation and thus a 3 -sum decomposition of $B$, or to conclude that no such decomposition is possible. PARTITION specifies that the smaller set $T$ of the input pair satisfies $|T| \geq 3$, but with a trivial modification the algorithm works just as well for the case $|T|=2$ arising here from deficient 3-separations.

Suppose a 3 -sum decomposition is carried out. In the original method, work done prior to that decomposition is used when component matrices are processed. This approach necessitates that tests for graphicness are accompanied with a search for decompositions. The implemented method avoids the complexity of that approach and simply proceeds recursively after each decomposition. In the simplified process, a sequence of nested 3-connected submatrices $N^{1}, \ldots, N^{k}=$ $B$ is determined, and a test of graphicness and cographicness is carried out for each matrix of the sequence until either $B$ is determined to be graphic or cographic, or a nongraphic and noncographic $N^{j+1}$ is found. In the latter case, the above-described search for a 3-sum decomposition either produces such a decomposition or results in the conclusion that the matrix is not regular.

We turn to the problem of signing the original $B$. The original method does such signing and then attempts to scale the signed version so that it becomes the input matrix $A$. In the implemented method, the signing of $A$ is taken into account when $B$ is signed, and thus the scaling step is not needed. Also, the signing is done at the very beginning since it is quickly done and may already determine the input matrix $A$ to be non-t.u.

The overall run time of the implemented method is $O\left((m+n)^{5}\right)$, since (1) PARTITION has at most quadratic run time, (2) a given nested sequence of 3 -connected matrices produces at most a quadratic number of input pairs for PARTITION, (3) there are at most a linear number of 3 -sum decompositions, and (4) the remaining steps are easily done in $O\left((m+n)^{5}\right)$ time.

The user might want to obtain a certificate together with the answer. A positive certificate consists of a tree whose inner nodes correspond to 1-, 2-, and 3 -sum decompositions, and whose end nodes correspond to graphs and copies of $B^{10.1}$ and $B^{10.1}$.

A negative certificate consists of a square matrix whose determinant has absolute value of at least 2. More interesting is a minimal violator that is not t.u., but all of whose proper submatrices are t.u. A simple strategy for finding a minimum violator recursively removes a single row or column, tests for total unimodularity, and adds the row or column back in if the submatrix turns out 
to be t.u. Three ideas reduce the number of total unimodularity tests during the search.

First, if the signing process determines $A$ to be non-t.u. while $B$ was found to be regular, then the signing directly determines a minimal violator having exactly two nonzeros in each row and column; see Section 5.

Second, suppose that the method has stopped since the currently processed matrix is nonregular. Due to the structure of the decompositions, that nonregular matrix is obtainable from the original matrix $B$ by a sequence of pivots followed by deletion of some rows and columns. The sequence of pivots and the deletions are readily determined. If a deleted row or column was never involved in a pivot, then its deletion from $B$ produces a smaller nonregular matrix. Hence, we carry out all such deletions.

The third idea is based on the fact that the given matrix may contain a number of minimal violators. This need not be so, as shown by the construction of [18] where the matrices having exactly one minimal violator are produced. Guessing that a number of minimal violators are present, we remove $80 \%$ of the rows or columns of the nonregular $B$. If the resulting submatrix is nonregular, recursion is used. Otherwise, we go back to the original matrix and remove $40 \%$ of the rows or columns. We go on by always halving the amount until we reach a fixed threshold. If the heuristic fails to produce a nonregular submatrix, we apply the naïve strategy and remove only a single row or column at a time.

We use a straightforward matrix implementation with $O(1)$ indexed access. For conceptual simplicity, subroutines sometimes move certain submatrices of a given matrix to the top left corner or carry out column/row permutations or transposition. For efficient handling of these cases, we have implemented various matrix proxies. The most important one is an object that refers to another matrix, but applies row and column permutations beforehand. With the help of generic programming, it can be used as a usual matrix, but only needs $O(1)$ time to swap two rows or columns.

Below, the implemented method is called Decomposition Test, for short DT. Method DT followed by construction of a minimal violator, if applicable, is called DT\&V.

\section{Enumerative Methods}

We also have implemented two naïve enumerative tests for comparison purposes. The first one tests the square submatrices using the criterion of Camion [4], according to which a matrix $A$ is t.u. if and only if, for every square submatrix $A^{\prime}$ of $A$ with even row and column sums, the sum of the entries of $A^{\prime}$ is divisible by 4 . Submatrix Test, for short ST, applies that test to the square submatrices of $A$ in increasing order.

The second one is based on the characterization of total unimodularity by Ghouila-Houri [8], which says that a matrix $A$ is t.u. if and only if for each column submatrix $A^{\prime}$ of $A$, there is a $\{ \pm 1\}$ vector $x$ such that $A^{\prime} x$ is a $\{0, \pm 1\}$ 
vector. Algorithm Column Enumeration, for short CE, carries out that test in straightforward fashion.

\section{Computational Results}

We have implemented DT, DT\&V, ST, and CE in C++ and have applied them to three matrix classes using an AMD Opteron with $2.3 \mathrm{GHz}$. In the tables below, the run time is measured in seconds and omitted if less than $0.1 \mathrm{sec}$.

The first class consists of randomly generated matrices. If the generation is carried out without some care, then the resulting matrices most likely contain a non-t.u. $2 \times 2$ submatrix and typically are detected to be non-t.u. by the signing process. Thus, the methods ST, CE, and DT would settle the cases very quickly. To prevent that trivial outcome, we generate $\{0,1\}$ matrices $B$ randomly and apply the signing procedure to obtain $\{0, \pm 1\}$ matrices $A$ which are then tested. The net effect of this change is that (1) ST and CE are less likely to terminate due to a $2 \times 2$ non-t.u. submatrix, and (2) DT and DT\&V never detect non-total unimodularity in the signing process and thus always carry out the generally difficult regularity test of $B$.

Details of the generation of the random matrices are as follows. For each $p=2 / 3,1 / 2,1 / 4$, and $1 / 8$, and each $n=200,400$, and 800, we randomly select ten $n \times n\{0,1\}$ matrices $B$ where $p$ is the probability that a given entry receives the value 1 . Then we apply the signing procedure to each $B$ to obtain $\{0, \pm 1\}$ matrices $A$. Thus, $A$ is t.u. if and only if $B$ is regular. To each such matrix $A$, the methods ST, CE, DT, and DT\&V are applied. In Table1 below, the results listed for each pair of $p$ and $n$ are the geometric means of the run times for the ten matrices.

Table 1. Running times for random matrices

\begin{tabular}{|c|c|r|r|r|r|}
\hline Size & $p$ & \multicolumn{1}{|c|}{ ST } & CE & DT & DT\&V \\
\hline $200 \times 200$ & $2 / 3$ & 185.4 & & & 0.1 \\
$400 \times 400$ & $2 / 3$ & 2948.8 & 0.1 & 0.3 & 0.4 \\
$800 \times 800$ & $2 / 3$ & $>3600$ & 0.2 & 0.7 & 0.9 \\
\hline $200 \times 200$ & $1 / 2$ & 28.2 & & & \\
$400 \times 400$ & $1 / 2$ & 218.3 & 0.1 & 0.1 & 0.1 \\
$800 \times 800$ & $1 / 2$ & $>3600$ & 0.3 & 0.7 & 0.7 \\
\hline $200 \times 200$ & $1 / 4$ & 0.1 & & 0.1 & 0.1 \\
$400 \times 400$ & $1 / 4$ & 0.2 & 0.1 & 0.3 & 0.3 \\
$800 \times 800$ & $1 / 4$ & 1.0 & 0.3 & 0.4 & 0.5 \\
\hline $200 \times 200$ & $1 / 8$ & 0.1 & & 0.1 & 0.1 \\
$400 \times 400$ & $1 / 8$ & 0.2 & 0.1 & 0.2 & 0.3 \\
$800 \times 800$ & $1 / 8$ & 0.5 & 0.2 & 0.6 & 2.0 \\
\hline
\end{tabular}

It turns out that all matrices are non-t.u., which is no surprise. The impressive performance of method $\mathrm{CE}$ is due to the fact that non-total unimodularity can 
be proved using few columns. Method DT also handles all cases well since it typically finds small 3-connected nongraphic and noncographic submatrices that result in few candidate pairs for induced (3|4)-separations. Method ST is third in performance and works well except for the cases with $p=2 / 3$ and $n=400,800$. The methods DT and DT\&V have similar run times. This is due to the fact that the heuristic for finding minimal violators described in Section 2.3 is very effective for the matrix class constructed here.

The second set of test matrices is generated from randomly generated directed networks. According to Theorem 2 these matrices and their transposes are in some sense the main building blocks of t.u. matrices. We generate the networks by constructing Erdös-Rényi graphs $G(n, p)$ (see [9]) and compute matrices representing the corresponding graphic matroids. The parameters $n$ and $p$ are chosen such that the resulting matrix is connected and of suitable size. For each matrix size listed below, we generate one instance. These matrices are t.u. and therefore should be difficult for the enumerative methods ST and CE. At the same time, DT should perform well since the method never needs to find a 3 -sum decomposition or locate a minimal violator. The data of Table 2 support these predictions.

Table 2. Running times for network matrices

\begin{tabular}{|c|r|r|r|}
\hline Size & \multicolumn{1}{|c|}{ ST } & CE & DT \\
\hline $10 \times 10$ & 0.3 & & \\
$12 \times 12$ & 1.8 & & \\
$14 \times 14$ & 21.6 & 0.1 & \\
$16 \times 16$ & 311.8 & 1.0 & \\
$18 \times 18$ & $>3600$ & 8.3 & \\
$20 \times 20$ & $>3600$ & 83.1 & \\
$22 \times 22$ & $>3600$ & 709.6 & \\
$24 \times 24$ & $>3600$ & $>3600$ & \\
$50 \times 50$ & $>3600$ & $>3600$ & \\
$100 \times 100$ & $>3600$ & $>3600$ & \\
$200 \times 200$ & $>3600$ & $>3600$ & 0.1 \\
$400 \times 400$ & $>3600$ & $>3600$ & 0.5 \\
$800 \times 800$ & $>3600$ & $>3600$ & 5.3 \\
\hline
\end{tabular}

The third class consists of matrices that contain exactly one square submatrix whose determinant is not equal to 0 or \pm 1 and thus must be a minimal violator. Each matrix is constructed from a square matrix of odd order $n \geq 5$ where each row and each column has exactly two 1s, arranged in cycle fashion. In that matrix, two adjacent rows are selected, and for any column where those two rows have $0 \mathrm{~s}$, both $0 \mathrm{~s}$ are replaced by $1 \mathrm{~s}$. It is readily shown that any such matrix is a minimal violator. Using results of [18, the matrix is transformed by pivots to one having exactly one violator of order $(n-1) / 2$. Thus, as $n$ grows, effort of ST and CE must grow exponentially, and even for modest values of $n$ the two methods 
should be unable to decide total unimodularity. On the other hand, DT should be able to process these difficult cases with reasonable efficiency. Finally, DT\&V should require significant additional effort beyond that for DT since each matrix has just one minimal violator, and that submatrix is relatively large. Table 3 confirms these predictions.

Table 3. Running times for odd-cycle matrices

\begin{tabular}{|r|r|r|r|r|}
\hline Size & \multicolumn{1}{|c|}{ ST } & CE & \multicolumn{1}{c|}{ DT } & DT\&V \\
\hline $11 \times 11$ & 0.7 & & & \\
$13 \times 13$ & 5.7 & & & \\
$15 \times 15$ & 79.3 & 0.6 & & \\
$17 \times 17$ & 1201.8 & 6.0 & & 0.1 \\
$19 \times 19$ & $>3600$ & 59.1 & & 0.1 \\
$21 \times 21$ & $>3600$ & 569.2 & & 0.2 \\
$23 \times 23$ & $>3600$ & $>3600$ & & 0.3 \\
$51 \times 51$ & $>3600$ & $>3600$ & 0.6 & 3.2 \\
$101 \times 101$ & $>3600$ & $>3600$ & 3.4 & 170.7 \\
$151 \times 151$ & $>3600$ & $>3600$ & 13.1 & 1302.5 \\
$201 \times 201$ & $>3600$ & $>3600$ & 32.1 & 3490.1 \\
$301 \times 301$ & $>3600$ & $>3600$ & 137.2 & $>3600$ \\
$401 \times 401$ & $>3600$ & $>3600$ & 476.3 & $>3600$ \\
$501 \times 501$ & $>3600$ & $>3600$ & 1457.1 & $>3600$ \\
$601 \times 601$ & $>3600$ & $>3600$ & 2422.0 & $>3600$ \\
$701 \times 701$ & $>3600$ & $>3600$ & $>3600$ & $>3600$ \\
\hline
\end{tabular}

\section{Details of Implementation}

This section provides implementation details. We skip the trivial enumerative tests ST and CE, and also omit steps that are identical to their counterparts in the original method. As an easy means of differentiation, we use the names of the procedures of the original method in capital letters, just as done in [17, and employ lower-case names for the subroutines introduced here. Bold fonts for both types of names help set them apart from the text.

The discussion proceeds in a top-down manner. Thus, we start with the main routine, which tests for total unimodularity.

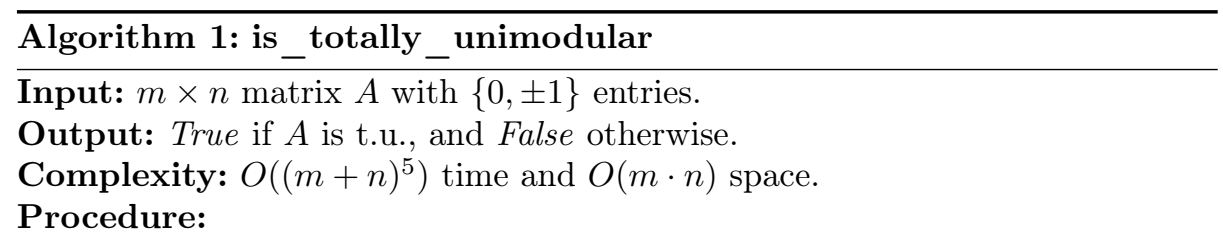

1. Call sign_matrix with $A$ as input. If the subroutine declares that $A$ has been modified, return False. 
2. Call decompose_matrix with the binary version $B$ of $A$ and an empty sequence of nested 3 -connected submatrices as input. Return the True/False output of the subroutine.

The signing procedure described next recursively modifies the entries of the $\{0, \pm 1\}$ input matrix $A$ so that certain minimal submatrices $V$ with exactly two nonzeros in each row and column and with entries summing to $2(\bmod 4)$ have the entries sum to $0(\bmod 4)$ after the signing. In the context of the main routine is_totally_unimodular, the matrix $A$ is t.u. if and only if the signing routine does not change any entry of $A$ and the binary version $B$ of $A$ is regular. On the other hand, if the signing routine does change at least one such entry, then the subroutine can be stopped when the first such change is to be made. The matrix $V$ on hand at that time is a minimal non-t.u matrix.

Recall that the signing procedure is used in the construction of the first class of test matrices, where each randomly generated matrix $B$ is signed by the signing procedure to obtain a matrix $A$. When methods DT and DT\&V later process each such test matrix $A$, the signing procedure does not alter $A$, and thus regularity of $B$ is tested. The outcome of the latter test then decides whether $A$ is t.u.

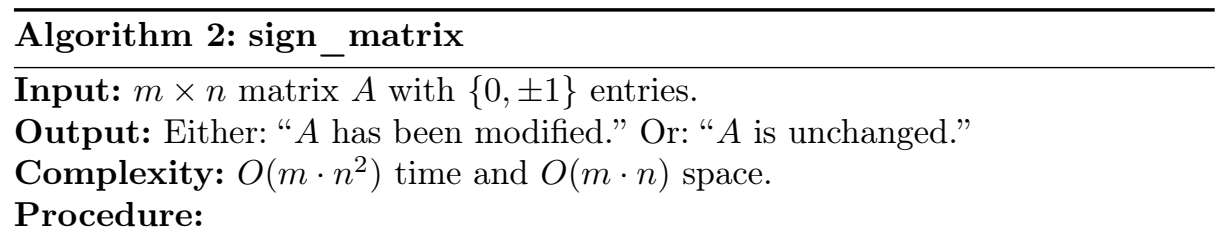

1. If $A$ is a zero matrix, output " $A$ is unchanged", and stop. Otherwise, let $y$ be the index of an arbitrarily selected nonzero column of $A$. Define $X$ to be the index set of the rows of $A$ containing the nonzeros of the selected column, and initialize $Y=\{y\}$.

2. If $Y$ is equal to the column index set of $A$, output " $A$ has been modified" if during any iteration in Step 4 an entry was changed, and output " $A$ is unchanged" otherwise; stop.

3. If there is a column index $y \notin Y$ for which the column vector $A_{X, y}$ is nonzero, select one such $y$, and go to Step 4. Otherwise, select any $y \notin Y$, and go to Step 5.

4. Let $A_{x_{j}, y}, j=0, \ldots, s$, be the nonzero entries of the column vector $A_{X, y}$. If the vector has just one nonzero entry and thus $s=0$, go to Step 5 . Otherwise, do a breadth-first-search on $B G\left(A_{X, Y}\right)$ to find shortest paths from $x_{0}$ to every other $x_{j}, j=1, \ldots, s$.

For $i=1, \ldots, s$, do the following steps. Going from $x_{i}$ to $x_{0}$ along the given shortest path, let the first $x_{j}, j \neq i$ encountered have index $j=p_{i}$. The path segment from $x_{p_{i}}$ to $x_{i}$ together with the edges $\left(x_{i}, y\right)$ and $\left(x_{p_{i}}, y\right)$ form a chordless cycle. In $A$, that cycle corresponds to a square submatrix $V$ with exactly two nonzero entries in each row and column. If the entries of that submatrix sum to $2(\bmod 4)$, flip the sign of $A_{x_{j}, y}$. 
5. Add $y$ to $Y$. Add the row indices $x$ for which $A_{x, y} \neq 0$ to $X$. Go to Step 2 .

Remark: Every iteration for given index $y$ is done in $O(m \cdot n)$ time.

The next subroutine is the top procedure for the regularity test of $B$. Given $B$, either (1) the subroutine decomposes $B$ in a 1- or 2-sum decomposition and then invokes recursion for the components; or (2) it determines $B$ to be isomorphic to $B^{10.1}$ or $B^{10.2}$ of Fig. 6 and thus to be regular; or (3) it constructs a sequence of nested 3 -connected submatrices $N^{1}, \ldots, N^{k}$ which are tested for graphicness and cographicness; if all submatrices turn out to be graphic or cographic, $B$ is regular; or (4) it uses the sequence $N^{1}, \ldots, N^{k}$ to decompose $B$ in a 3 -sum decomposition and then invokes recursion for the components of the decomposition; or, if none of (1)-(4) apply, (5) it concludes that $B$ is not regular.

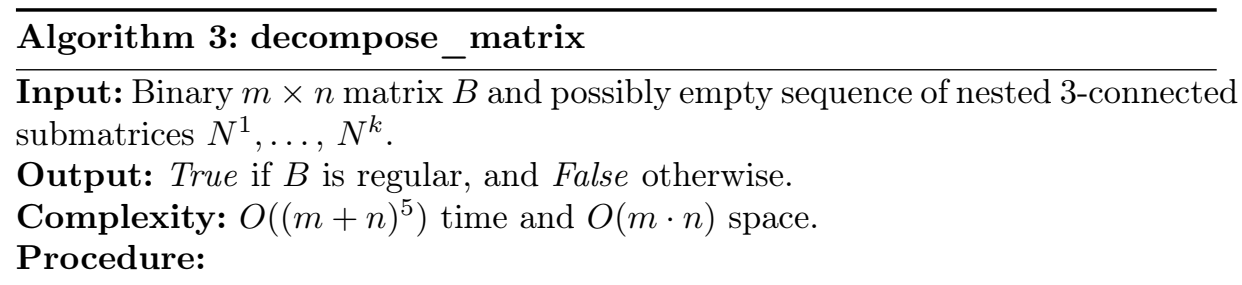

1. If $m<3$ or $n<3$, then $B$ is regular; return True, and stop.

2. If the input sequence of nested 3-connected submatrices is nonempty, go to Step 4. Otherwise, go to Step 3.

3. Call FIND-B. If it finds a 1- or 2-separation, decompose $B$ into $B_{1}$ and $B_{2}$ according to the separation, and call decompose matrix with each of them and the empty sequence as input. Return True if both calls return True, return False otherwise, and stop.

If FIND-B does not find a 1- or 2-separation, it has identified a submatrix $N^{1}$ that represents the graph $W_{3}$. Initialize $k=1$, and go to Step 4 .

4. If $N^{k}=B$, go to Step 5. Otherwise, call EXTEND-B, and try to find a submatrix $N^{k+1}$ of $B$ which contains $N^{k}$.

If EXTEND-B succeeds, increment $k$ by 1 , and repeat this step. Otherwise, the subroutine returns a 1 - or 2 -separation of $B$ into $B_{1}$ and $B_{2}$ where $B_{1}$ contains a 3 -connected submatrix sequence that is isomorphic to $N^{1}, \ldots$, $N^{k}$. Relabel that sequence as $N^{1}, \ldots, N^{k}$. Call decompose matrix twice: once with $B_{1}$ and $N^{1}, \ldots, N^{k}$, and the second time with $B_{2}^{-}$and the empty sequence. Return True if both calls return True, return False otherwise, and stop.

5. If $B$ is $5 \times 5$, test whether $B G(B)$ is isomorphic to $B G\left(B^{10.1}\right)$ or $B G\left(B^{10.2}\right)$, where $B^{10.1}$ and $B^{10.2}$ are shown in Fig. 6 . The isomorphism test is trivial due to the size and special structure of $B^{10.1}$ and $B^{10.2}$. In the affirmative case, return True, and stop.

6. Call test_graphicness to decide graphicness of $B$ via the sequence $N^{1}, \ldots$, $N^{k}$. If the output is " $B$ is graphic," return True, and stop. Otherwise, call 
test_graphicness once more to decide graphicness of $B^{t}$ via $N^{1^{t}}, \ldots, N^{k^{t}}$. If the output is " $B^{t}$ is graphic," return True, and stop.

7. In Step 6, each of the two calls of test_graphicness returned an index. Let $j$ the larger of the two returned indices. Thus, $N^{1}, \ldots, N^{j}$ are graphic or cographic, while $N^{j+1}$ is not graphic and not cographic.

For every $i=1, \ldots, j$, define pairs $\left(T, E^{i} \backslash T\right)$ corresponding to possibly deficient 3-separations of $N^{i}$, as stated in Section 2.3. For each such pair, call PARTITION to test whether the pair induces a $(3 \mid 4)$-separation of $B$. As soon as an induced decomposition of $B$ is detected, say into $B_{1}$ and $B_{2}$, call decompose_matrix twice, once with $B^{1}$ and the empty sequence, and the second time with $B^{2}$ and the empty sequence. Return True if both calls return True, return False otherwise, and stop.

If none of the pairs defined for the matrices $N^{1}, \ldots, N^{j}$ induces a $(3 \mid 4)$ separation of $B$, return False, and stop.

Remark: The implementations of FIND-B and EXTEND-B run in $O((\mathrm{~m}+$ $n)^{3}$ ) time, while that of PARTITION runs in $O(m \cdot n)$ time.

Subroutine test_graphicness is described next. It calls TEST-C repeatedly to extend the graph of a submatrix $N^{i}$ to the graph of the next larger submatrix $N^{i+1}$. TEST-C contains a minor error arising from two special cases. The code uses the amended version.

\footnotetext{
Algorithm 4: test_graphicness

Input: Binary $m \times n$ matrix $B$ and nonempty sequence $N^{1}, \ldots, N^{k}=B$ of nested 3-connected submatrices.

Output: Either: " $B$ is graphic" together with the corresponding graph $G$. Or: " $B$ is not graphic" and the index of the largest graphic $N^{i}$.

Complexity: $O\left((m+n)^{3}\right)$ time and $O(m \cdot n)$ space.

Procedure:

1. Define $G^{1}$ to be the wheel graph $W_{3}$, which represents $N^{1}$.

2. For $i=1, \ldots, k-1$, call TEST-C to attempt extension of the graph $G^{i}$ for $N^{i}$ to a graph $G^{i+1}$ for $N^{i+1}$. If such an extension is not possible for some $N^{i}$, return " $B$ is not graphic" and index $i$, and stop. Otherwise, return " $B$ is graphic" together with the graph $G^{k}$ for $N^{k}$, and stop.

Remark: The implementation of TEST-C runs in $O(m \cdot n)$.

If $A$ is not t.u., a minimal violator is found as described in Section 2.3. Finally, tests for unimodularity and strong unimodularity have been implemented in straightforward fashion using Theorem 1 . In the unimodularity test of an integer matrix $A$, Gaussian elimination selects a basis $C$ of $A$ and solves the equation $C X=A$ for $X$. Computation of the Smith Normal Form 13. settles whether $\operatorname{gcd}_{i} \operatorname{det} C^{i}=1$, and is_totally_unimodular decides if $X$ is t.u. The matrix $A$ is unimodular if and only if $\operatorname{gcd}_{i} \operatorname{det} C^{i}=1$ and $X$ is t.u. Strong unimodularity of $A$ is decided by testing if both $A$ and $A^{t}$ are unimodular.
} 


\section{Summary}

The work reported here was motivated by the practical need for an effective computer program testing total unimodularity, strong unimodularity, and unimodularity. We decided to use the algorithm of [17] for the total unimodularity test, which for $m \times n$ matrices has $O\left((m+n)^{3}\right)$ complexity. Exact implementation of that algorithm is rather daunting, so we used a somewhat simplified version with $O\left((m+n)^{5}\right)$ complexity. Computational tests indicate that matrices with several hundred rows and columns should be handled in reasonable time. For the processing of larger matrices, the implementation could be refined using the parts of the algorithm of [17] that have been simplified here. An intermediate remedy could also be implemented where the time-consuming search for decompositions is handled by parallel processors. The present program is purposely structured to simplify such a shift to parallel computation.

\section{References}

1. R. E. Bixby and W. H. Cunningham. Converting linear programs to network problems. Math. Op. Res., 5:321-357, 1980.

2. R. E. Bixby, W. H. Cunningham, and R. Rajan. A decomposition algorithm for matroids. Technical report, Rice University, 1986.

3. Boost Software License http://www . boost.org/LICENSE_1_0.txt.

4. P. Camion. Matrices Totalement Unimodulaire et Problèmes Combinatoires. PhD thesis, Université Libre de Bruxelles, Bruxelles, 1963.

5. W. H. Cunningham and J. Edmonds. Decomposition of linear systems, (unpublished). 1965.

6. J. Edmonds. Minimum partition of a matroid into independet subsets. J. Res. Nat. Bur. Std. (B), 69:67-72, 1965.

7. S. Fujishige. An efficient $p q$-graph algorithm for solving the graph-realization problem. J. Computer and Systems Sciences, 21:63-86, 1980.

8. A. Ghouila-Houri. Caracterisation des matrices totalement unimodulaires. C.R. Acad. Sci. Paris, 254:1192-1194, 1962.

9. E. N. Gilbert. Random graphs. Annals of Mathematical Statistics, 30:1141-1144, 1959.

10. A. J. Hoffman and J. B. Kruskal. Integral boundary points of convex polyhedra. Annals Math. Studies, 38:223-246, 1956.

11. A. J. Hoffman and R. Oppenheim. Local unimodularity in the matching polytope. Annals of Discrete Mathematics, 2:201-209, 1978.

12. P. D. Seymour. Decomposition of regular matroids. J. Comb. Theory, Ser. B, 28:305-359, 1980.

13. H. J. S. Smith. On systems of linear indeterminate equations and congruences. Philos. Trans. Roy. Soc. London, 151:293-326, 1861-1862.

14. Software available at: http://www.utdallas.edu/ /klaus/TUtest/ http://www.math. uni-magdeburg.de/ / walter/TUtest/.

15. K. Truemper. Algebraic characterizations of unimodular matrices. SIAM J. Appl. Math., 35:328-332, 1978. 
16. K. Truemper. Complement total unimodularity. Lin. Alg. Appl., 30:77-92, 1980.

17. K. Truemper. A decomposition theory for matroids. V. Testing of matrix total unimodularity. J. Comb. Theory, Ser. B, 49:241-281, 1990.

18. K. Truemper. A decomposition theory for matroids. VII. Analysis of minimal violation matrices. J. Comb. Theory, Ser. B, 55:302-335, 1992.

19. K. Truemper. Matroid Decomposition (Revised Edition). Leibniz Company, 1998.

20. K. Truemper and R. Chandrasekaran. Local unimodularity of matrix-vector pairs. Lin. Alg. Appl., 22:65-78, 1978.

21. W. T. Tutte. A homotopy theorem for matroids i, ii. Trans. Amer. Math. Soc., 88:527-552, 1958.

22. H. Whitney. 2-isomorphic graphs. Am. J. Math., 55:245-254, 1933. 